\title{
ON CLASSES OF MEROMORPHIC LOCALLY UNIVALENT FUNCTIONS DEFINED BY DIFFERENTIAL INEQUALITIES
}

\author{
SEE KEONG LEE, SAMINATHAN PONNUSAMY, AND KARL-JOACHIM WIRTHS
}

\begin{abstract}
In this article we consider functions meromorphic in the unit disk. We give an elementary proof for a condition that is sufficient for the univalence of such functions which also contains some known results. We include few open problems for further research.
\end{abstract}

\section{Preliminaries and Main Results}

We denote the unit disk by $\mathbb{D}=\{z \in \mathbb{C}:|z|<1\}$ and let

$$
\mathcal{A}=\left\{f: \quad f \text { is analytic in } \mathbb{D}, f(0)=f^{\prime}(0)-1=0\right\} .
$$

The family $\mathcal{S}$ of univalent functions in $\mathcal{A}$ together with its many subfamilies, for which the image domains have special geometric properties, have been investigated in details. See $[3,4]$. Throughout, $\mathcal{B}$ denotes the class of functions $\omega$, analytic in $\mathbb{D}$ such that $|\omega(z)| \leq 1$ for $z \in \mathbb{D}$. The well known inequality $\sum_{k=0}^{\infty}\left|c_{k}\right|^{2} \leq 1$ for $\Omega(z)=\sum_{k=0}^{\infty} c_{k} z^{k} \in \mathcal{B}$ will be used to get the proof of Theorem 4. Recently, in $[8$, Theorem 2(b)], the second and the third authors proved among other things the following result which extends the earlier known result for analytic functions.

Theorem A. Let $f$ be meromorphic in $\mathbb{D}$ such that $f(0)=f^{\prime}(0)-1=0$. If for all $z \in \mathbb{D}$ the inequality

$$
\left|\frac{z}{f(z)}-z\left(\frac{z}{f(z)}\right)^{\prime}-1\right|<\lambda, z \in \mathbb{D}
$$

is valid for some $\lambda \in(0,1]$, then $f$ is univalent in $\mathbb{D}$.

The proof of Theorem A in [8] was elegant and was also different from the other known methods. In this article, we shall consider slightly more general situation. For $0<\lambda$ and $\mu \in \mathbb{C}$ such that $|1-\mu|<\lambda$, we consider the family $\mathcal{U}(\lambda, \mu)$ of meromorphic functions $f$ satisfying the inequality

$$
\left|U_{f}(z)-\mu\right|<\lambda \text { in } \mathbb{D}
$$

where

$$
U_{f}(z):=\left(\frac{z}{f(z)}\right)^{2} f^{\prime}(z)=\frac{z}{f(z)}-z\left(\frac{z}{f(z)}\right)^{\prime}, \quad z \in \mathbb{D} .
$$

2010 Mathematics Subject Classification. 30C45.

Key words and phrases. Meromorphic univalent functions, subordination, coefficient estimates

File: arXiv`PoWirths3²017`.tex, printed: 7-5-2019, 2.09. 
Note that the center 1 has been replaced by $\mu$. First we consider the problem of determining conditions on $\lambda$ and $\mu$ so that functions in $\mathcal{U}(\lambda, \mu)$ are univalent in $\mathbb{D}$.

As with the case of analytic functions, for notational simplicity, we let $\mathcal{U}(\lambda):=$ $\mathcal{U}(\lambda, 1), \mathcal{U}:=\mathcal{U}(1)$. In the analytic case, it was well-known that $\mathcal{U} \subsetneq \mathcal{S}$ (see $[1,2])$.

Theorem 1. Let $\lambda \in(0,1]$ and $|1-\mu|<\lambda$. All members of the family $\mathcal{U}(\lambda, \mu)$ are functions meromorphic and locally univalent in $\mathbb{D}$ if and only if $|\mu| \geq \lambda$.

Proof. It is a simple exercise to see that if $f \in \mathcal{U}(\lambda, \mu)$, then $f(z) \neq 0$ in $\mathbb{D} \backslash\{0\}$, because otherwise $f\left(z_{0}\right)=0$ for some $z_{0} \in \mathbb{D} \backslash\{0\}$ which would then imply that

$$
f(z)=c_{m}\left(z-z_{0}\right)^{m}+\cdots \quad\left(c_{m} \neq 0, m \geq 1\right)
$$

in a neighborhood of $z_{0}$ so that

$$
U_{f}(z)=\frac{m z_{0}}{c_{m}\left(z-z_{0}\right)^{m+1}}+\cdots
$$

implying that $U_{f}(z)$ has a pole of order $m+1$ which would clearly be a contradiction to the fact that $U_{f}(z)$ is bounded. Moreover, for $|\mu| \geq \lambda$, the inequality $\left|U_{f}(z)-\mu\right|<$ $\lambda$ for $z \in \mathbb{D}$ implies $\left|U_{f}(z)\right|>0$ for $z \in \mathbb{D}$ and hence, in either way $f^{\prime}(z) \neq 0$ for $z \in \mathbb{D}$ and $f(z) \neq 0$ in $\mathbb{D} \backslash\{0\}$.

To prove the other direction of our assertion, we let $f(z)=z+\sum_{n=2}^{\infty} a_{n} z^{n}$ in $\mathcal{U}(\lambda, \mu)$ and consider

$$
\lambda \Omega(z)=\frac{z}{f(z)}-z\left(\frac{z}{f(z)}\right)^{\prime}-\mu=1-\mu+\left(a_{3}-a_{2}^{2}\right) z^{2}+\cdots=1-\mu+O\left(z^{2}\right)
$$

as $z \rightarrow 0$, where $\Omega$ is analytic in $\mathbb{D}$ such that $\Omega(0)=(1-\mu) / \lambda, \Omega^{\prime}(0)=0$ and $|\Omega(z)|<1$ in $\mathbb{D}$. For simplicity, we let $\Omega(0)=a$. Then

$$
\phi(z)=\frac{\Omega(z)-a}{1-\bar{a} \Omega(z)}
$$

is a Schwarz function, i. e., $\phi(0)=0$ and $|\phi(z)|<1$ for $z \in \mathbb{D}$. Hence,

$$
\Omega(z)=\frac{\phi(z)+a}{1+\bar{a} \phi(z)} \text {. }
$$

Since $\Omega^{\prime}(0)=\left(1-|a|^{2}\right) \phi^{\prime}(0)=0$, we have $\phi^{\prime}(0)=0$. Therefore, we can write $\phi(z)=z^{2} \omega(z)$, where $\omega \in \mathcal{B}$. Consequently,

$$
\frac{z}{f(z)}-z\left(\frac{z}{f(z)}\right)^{\prime}-\mu=\lambda\left(\frac{a+z^{2} \omega(z)}{1+\bar{a} z^{2} \omega(z)}\right), \quad z \in \mathbb{D},
$$

where $a=(1-\mu) / \lambda$ and $\omega \in \mathcal{B}$. Note that, in the neighborhood of $z=0$, we have the representation

$$
\begin{aligned}
\frac{z}{f(z)} & =\frac{1}{1+\sum_{n=2}^{\infty} a_{n} z^{n-1}} \\
& =1-\left(\sum_{n=2}^{\infty} a_{n} z^{n-1}\right)+\left(\sum_{n=2}^{\infty} a_{n} z^{n-1}\right)^{2}+\cdots \\
& =1-a_{2} z+\left(a_{2}^{2}-a_{3}\right) z^{2}+\cdots
\end{aligned}
$$


As with the standard procedure, the integration of the differential equation (3) delivers that each $f \in \mathcal{U}(\lambda, \mu)$ has the representation

$$
f(z)=\frac{z}{1+c z-\lambda z \int_{0}^{z} \frac{\left(1-|a|^{2}\right) \omega(t)}{1+\bar{a} t^{2} \omega(t)} d t},
$$

where $c=-a_{2}, a=\frac{1-\mu}{\lambda}$ and $\omega \in \mathcal{B}$. Now, we let $\omega(z)=1, z \in \mathbb{D}$, in this representation and obtain that

$$
f(z)=\frac{z}{1+c z-\lambda z \int_{0}^{z} \frac{\left(1-|a|^{2}\right)}{1+\bar{a} t^{2}} d t} .
$$

It follows that there exists a $z_{1} \in \mathbb{D}$ such that $f^{\prime}\left(z_{1}\right)=0$ if and only if

$$
\frac{z_{1}^{2}}{1+\bar{a} z_{1}^{2}}=\frac{-1}{\lambda\left(1-|a|^{2}\right)} \text {. }
$$

This is equivalent to

$$
\left|\lambda\left(1-|a|^{2}\right)+\bar{a}\right|>1
$$

or equivalently $|\mu|<\lambda$, which is a contradiction to the local univalency of $f$. Hence, the rest of the assertion is proved.

Theorem 2. Let $f$ be meromorphic in $\mathbb{D}$ such that $f(0)=f^{\prime}(0)-1=0$. Then $f \in U(\lambda, \mu)$ is univalent in $\mathbb{D}$ if either (a) $|1-\mu|<\lambda \leq 1 / 2$ or (b) $\lambda \in(1 / 2,1]$ and $|1-\mu| \leq 1-\lambda$.

Proof. By using the representation (4), we can write $f=1 / g_{\omega}$ for $f \in \mathcal{U}(\lambda, \mu)$, where

$$
g_{\omega}(z)=\frac{1}{z}\left[1+c z-\lambda z \int_{0}^{z} \frac{\left(1-|a|^{2}\right) \omega(t)}{1+\bar{a} t^{2} \omega(t)} d t\right] .
$$

We see that we have to prove

$$
0 \neq \frac{g_{\omega}\left(z_{1}\right)-g_{\omega}\left(z_{2}\right)}{z_{1}-z_{2}}=\frac{-1}{z_{1} z_{2}}-\frac{\lambda}{z_{1}-z_{2}} \int_{z_{2}}^{z_{1}} \frac{\left(1-|a|^{2}\right) \omega(t)}{1+\bar{a} t^{2} \omega(t)} d t,
$$

where $z_{1}, z_{2} \in \mathbb{D} \backslash\{0\}, z_{1} \neq z_{2}$, and $\omega \in \mathcal{B}$. Since $a=(1-\mu) / \lambda$ and

$$
\left|\frac{\left(1-|a|^{2}\right) \omega(z)}{1+\bar{a} z^{2} \omega(z)}\right| \leq \frac{1-|a|^{2}}{1-|a|}=1+|a|, \quad z \in \mathbb{D},
$$

we get

$$
\left|\lambda \frac{z_{1} z_{2}}{z_{1}-z_{2}} \int_{z_{2}}^{z_{1}} \frac{\left(1-|a|^{2}\right) \omega(t)}{1+\bar{a} t^{2} \omega(t)} d t\right|<\lambda(1+|a|)=\lambda+|1-\mu|
$$

and thus, (5) holds whenever $|1-\mu| \leq 1-\lambda$. Hence, $f$ is univalent if $|1-\mu| \leq$ $1-\lambda$. Therefore, every $f \in \mathcal{U}(\lambda, \mu)$ is univalent in $\mathbb{D}$ whenever $|1-\mu|<\lambda \leq \frac{1}{2}$ or $|1-\mu| \leq 1-\lambda$ with $\lambda \in\left(\frac{1}{2}, 1\right]$. This completes the proof. 
Remark 1. By using Theorem 1, we see that at least for nonnegative real numbers $\mu$ the assertion of Theorem 2(b) is best possible. This follows from the fact that for $\mu \in(1-\lambda, \lambda)$, the family $\mathcal{U}(\lambda, \mu)$ contains a function that is not locally univalent in $\mathbb{D}$. In order to present a couple of precise functions, we consider the function $f_{0}$ defined by (see also Problem 2)

$$
\frac{z}{f_{0}(z)}=1-z+\frac{\lambda\left(1-a^{2}\right) z}{2 \sqrt{a}} \log \left(\frac{1-(\sqrt{a} /(1+\sqrt{a}))(1-z)}{1+(\sqrt{a} /(1-\sqrt{a}))(1-z)}\right), \quad a=\frac{1-\mu}{\lambda} .
$$

Problem 1. Do there exist families $\mathcal{U}(\lambda, \mu)$ consisting of univalent functions besides those mentioned in Theorem 2?

In the following we use the equation

$$
\frac{z}{f(z)}-z\left(\frac{z}{f(z)}\right)^{\prime}-\mu=\lambda \Omega(z)
$$

where

$$
\Omega(z)=\frac{1-\mu}{\lambda}+\sum_{k=2}^{\infty} c_{k} z^{k}
$$

with $\Omega \in \mathcal{B}$ to get sharp estimates for the coefficients of the representation

$$
\frac{z}{f(z)}=1+\sum_{k=1}^{\infty} b_{k} z^{k}=1-a_{2} z+\left(a_{2}^{2}-a_{3}\right) z^{2}+\cdots
$$

Theorem 3. For $f \in \mathcal{U}(\lambda, \mu)$ of the form (8) and $k \geq 2$, the inequalities

$$
\left|b_{k}\right| \leq \frac{\lambda}{k-1}\left(1-\frac{|1-\mu|^{2}}{\lambda^{2}}\right)
$$

are valid. These inequalities are best possible.

Proof. From (6) and (7) we derive the identities

$$
b_{k}(1-k)=\lambda c_{k}, \quad k \geq 2 .
$$

The well known inequalities $\left|c_{k}\right| \leq 1-\left|c_{0}\right|^{2}$ for $\Omega(z)=\sum_{k=0}^{\infty} c_{k} z^{k}$ with $\Omega \in \mathcal{B}$, $c_{0}=(1-\mu) / \lambda$ and $c_{1}=0$, imply the validity of our assertion.

For the proof of the sharpness, we set $\omega(z)=-z^{k-2}$ in (4) (with $c_{0}=a$ ) and consider the following functions $f_{k}$ for $k \geq 2$ :

$$
\frac{z}{f_{k}(z)}=1+c z+\lambda z \int_{0}^{z} \frac{\left(1-|a|^{2}\right) t^{k-2}}{1-\bar{a} t^{k}} d t=1+c z+\lambda\left(1-|a|^{2}\right) \sum_{j=2}^{\infty}(\bar{a})^{j} \frac{z^{(j+1) k}}{(j+1) k-1} .
$$

Obviously, $f_{k} \in \mathcal{U}(\lambda, \mu)$, and further we get that the $k$-th coefficient $b_{k}$ of the function $z / f_{k}(z)$ satisfies

$$
b_{k}=\frac{\lambda}{k-1}\left(1-\frac{|1-\mu|^{2}}{\lambda^{2}}\right) .
$$

The proof is complete. 
Remark 2. In particular, in the case $k=2$ of Theorem 3, we get the sharp inequality

$$
\left|b_{2}\right|=\left|a_{2}^{2}-a_{3}\right| \leq \lambda\left(1-\frac{|1-\mu|^{2}}{\lambda^{2}}\right)
$$

for $f(z)=z+\sum_{n=2}^{\infty} a_{n} z^{n} \in \mathcal{U}(\lambda, \mu)$.

The well known inequality $\sum_{k=0}^{\infty}\left|c_{k}\right|^{2} \leq 1$ for $\Omega(z)=\sum_{k=0}^{\infty} c_{k} z^{k} \in \mathcal{B}$ will be used to get the proof of Theorem 4 .

Theorem 4. If $f \in \mathcal{U}(\lambda, \mu)$ is of the form (8), then

$$
\sum_{k=2}^{\infty}\left|b_{k}\right|^{2}(k-1)^{2} \leq \lambda^{2}\left(1-\frac{|1-\mu|^{2}}{\lambda^{2}}\right) .
$$

This inequality is best possible.

Proof. In view of the relations (6) and (7), the assumption gives

$$
\left|1-\mu-\sum_{k=2}^{\infty}(k-1) b_{k} z^{k}\right| \leq \lambda, \quad z \in \mathbb{D},
$$

from which the desired inequality follows because $\sum_{k=0}^{\infty}\left|c_{k}\right|^{2} \leq 1$ for $\Omega(z)=\sum_{k=0}^{\infty} c_{k} z^{k} \in$ $\mathcal{B}$. Thus, it remains to prove the assertion of the sharpness. To that end, we consider the functions $f_{k}, k \geq 2$, given by (9). Then their Taylor expansions are given by

$$
\frac{z}{f_{k}(z)}=1+c z+\lambda\left(1-|a|^{2}\right) \sum_{j=0}^{\infty} \bar{a}^{j} \frac{z^{(j+1) k}}{(j+1) k-1} .
$$

Hence, in these cases we get

$$
\sum_{k=2}^{\infty}\left|b_{k}\right|^{2}(k-1)^{2}=\lambda^{2}\left(1-|a|^{2}\right)^{2} \sum_{j=0}^{\infty}|a|^{2 j}=\lambda^{2}\left(1-\frac{|1-\mu|^{2}}{\lambda^{2}}\right) .
$$

This completes the proof of the sharpness.

Finding sharp estimates for the Taylor coefficients of the functions in $U(\lambda, \mu)$ turned out to be a challenge. As a first result in this direction we prove the next result which extends [8, Theorem 4].

Theorem 5. If $f \in \mathcal{U}(\lambda, \mu)$ is analytic in the disk $\mathbb{D}_{p}=\{z:|z|<p\}, p \in(0,1]$, and $a=(1-\mu) / \lambda$, then the inequality

$$
\left|a_{2}\right| \leq A_{2}:=\frac{1}{p}+\lambda \int_{0}^{p} \frac{\left(1-|a|^{2}\right) d t}{1-|a| t^{2}}=\left\{\begin{array}{r}
\frac{1}{p}+\frac{\lambda\left(1-|a|^{2}\right)}{2 \sqrt{|a|}} \log \left(\frac{1+p \sqrt{|a|}}{1-p \sqrt{|a|}}\right) \\
\frac{1}{p}+\lambda p \text { for } a \neq 0
\end{array}\right.
$$

is valid. This estimate is best possible for $\mu \in(1-\lambda, 1]$. 
Proof. We assume on the contrary that $\left|a_{2}\right|>A_{2}$. In other words, we can assume that there exists an $r \in(0,1)$ such that

$$
\left|a_{2}\right|=\frac{A_{2}}{r}=\frac{1}{p r}\left(1+\lambda p \int_{0}^{p} \frac{\left(1-|a|^{2}\right) d t}{1-\left.|a|\right|^{2}}\right) .
$$

Using Brouwer's fixed point theorem, we shall prove that then the function

$$
f(z)=\frac{z}{1-a_{2} z-\lambda z \int_{0}^{z} \frac{\left(1-|a|^{2}\right) \omega(t)}{1+\bar{a} t^{2} \omega(t)} d t},
$$

has a pole in the disk $\overline{\mathbb{D}}_{r p}:=\{z:|z| \leq p r\}$. To that end, we consider the function

$$
F(z)=\frac{1}{a_{2}}\left(1-\lambda z \int_{0}^{z} \frac{\left(1-|a|^{2}\right) \omega(t)}{1+\bar{a} t^{2} \omega(t)} d t\right)
$$

and we show that it has a fixed point in the disk $\mathbb{D}_{p r}$. For $|z| \leq p r$, we get

$$
|F(z)| \leq \frac{\left(1+\lambda p r \int_{0}^{p r} \frac{\left(1-|a|^{2}\right) d t}{1-|a| t^{2}}\right) p r}{1+\lambda p \int_{0}^{p} \frac{\left(1-|a|^{2}\right) d t}{1-|a| t^{2}}}<p r .
$$

Since $F$ is a continuous function that maps the convex compact set $\overline{\mathbb{D}_{p r}}$ into itself, Brouwer's fixed point theorem implies that $F$ has a fixed point in $\overline{\mathbb{D}_{p r}}$ which is a contradiction to the initial assumptions of Theorem 5. Hence $\left|a_{2}\right| \leq A_{2}$ is valid.

Concerning the sharpness, we see that for the numbers $\mu$ in question, the quantity $(1-\mu) / \lambda$ is nonnegative. We choose $\omega(z)=-1$ in the representation formula for $f \in \mathcal{U}(\lambda, \mu)$, and we get that the function $f=f_{0}$, where

$$
f_{0}(z)=\frac{z}{1-z\left(\frac{1}{p}+\lambda \int_{0}^{p} \frac{\left(1-a^{2}\right) d t}{1-a t^{2}}\right)+\lambda z \int_{0}^{z} \frac{\left(1-a^{2}\right) d t}{1-a t^{2}}}, a=\frac{1-\mu}{\lambda},
$$

which is analytic in $\mathbb{D}_{p}$ and achieves equality in the estimate of our theorem.

Note that the function $f_{0}$ given by (10) takes the form

$$
\frac{z}{f_{0}(z)}=\left\{\begin{array}{cc}
1-z\left(\frac{1}{p}+\frac{\lambda\left(1-a^{2}\right)}{2 \sqrt{a}} \log \left(\frac{1+p \sqrt{a}}{1-p \sqrt{a}}\right)\right) & \\
+\frac{\lambda\left(1-a^{2}\right) z}{2 \sqrt{a}} \log \left(\frac{1+\sqrt{a} z}{1-\sqrt{a} z}\right) & \text { for } a \neq 0 \\
1-((1 / p)+\lambda) z+\lambda z^{2} & \text { for } a=0,
\end{array}\right.
$$

which may be simplified as

$$
\begin{aligned}
\frac{z}{f_{0}(z)} & = \begin{cases}1-\frac{z}{p}+\frac{\lambda\left(1-a^{2}\right) z}{2 \sqrt{a}} \log \left(\frac{1+\sqrt{a} z}{1-\sqrt{a} z} \frac{1-p \sqrt{a}}{1+p \sqrt{a}}\right) & \text { for } a \neq 0 \\
1-((1 / p)+\lambda) z+\lambda z^{2} & \text { for } a=0,\end{cases} \\
& = \begin{cases}1-z+\frac{\lambda\left(1-a^{2}\right) z}{2 \sqrt{a}} \log \left(\frac{1-(\sqrt{a} /(1+p \sqrt{a}))(p-z)}{1+(\sqrt{a} /(1-p \sqrt{a}))(p-z)}\right) & \text { for } a \neq 0 \\
1-((1 / p)+\lambda) z+\lambda z^{2} & \text { for } a=0 .\end{cases}
\end{aligned}
$$

In the case of $p=1$, we then ask in particular the following. 
Problem 2. Suppose that $f \in \mathcal{U}(\lambda, \mu)$ is analytic in the unit disk $\mathbb{D}$, and $a=$ $(1-\mu) / \lambda>0$. Is

$$
\frac{z}{f(z)} \prec \frac{z}{f_{0}(z)}=1-z+\frac{\lambda\left(1-a^{2}\right) z}{2 \sqrt{a}} \log \left(\frac{1-(\sqrt{a} /(1+\sqrt{a}))(1-z)}{1+(\sqrt{a} /(1-\sqrt{a}))(1-z)}\right) ?
$$

Note that this problem has been solved in [5] for the case of $a=0$, i.e. for $\mu=1$.

Remark 3. If $\mu \notin(1-\lambda, 1]$, it is possible to get an implicit sharp upper estimate for $\left|a_{2}\right|$ of functions $f \in \mathcal{U}(\lambda, \mu)$ analytic in the disk $\mathbb{D}_{p}$ in the following way: We consider again

$$
F(z)=\frac{1}{a_{2}}\left(1-\lambda z \int_{0}^{z} \frac{\left(1-|a|^{2}\right) \omega(t)}{1+\bar{a} t^{2} \omega(t)} d t\right)
$$

and assume that there exists a number $r<1$ such that

$$
\left|a_{2}\right|=\frac{1}{p r} \max \left\{\left|1-\lambda z \int_{0}^{z} \frac{\left(1-|a|^{2}\right) \omega(t)}{1+\bar{a} t^{2} \omega(t)} d t\right|:|z|=p, \omega \in \mathcal{B}\right\} .
$$

Then we use the continuity of the function $F$ on the disk $\overline{\mathbb{D}_{p r}}$, the inclusion $F\left(\overline{\mathbb{D}_{p r}}\right) \subset$ $\overline{\mathbb{D}_{p r}}$, and Brouwer's fixed point theorem to see that $F$ has a fixed point in $\overline{\mathbb{D}_{p r}}$. This contradicts the assumption that $f$ is analytic in the disk $\mathbb{D}_{p}$. Hence,

$$
\left|a_{2}\right| \leq \frac{1}{p} \max \left\{\left|1-\lambda z \int_{0}^{z} \frac{\left(1-|a|^{2}\right) \omega(t)}{1+\bar{a} t^{2} \omega(t)} d t\right|:|z|=p, \omega \in \mathcal{B}\right\} .
$$

To prove the sharpness of this inequality, we choose $z_{0},\left|z_{0}\right|=p$, and $\omega_{0} \in \mathcal{B}$ such that

$$
\begin{aligned}
\mid 1-\lambda z_{0} \int_{0}^{z_{0}} & \frac{\left(1-|a|^{2}\right) \omega_{0}(t)}{1+\bar{a} t^{2} \omega_{0}(t)} d t \mid= \\
& \max \left\{\left|1-\lambda z \int_{0}^{z} \frac{\left(1-|a|^{2}\right) \omega(t)}{1+\bar{a} t^{2} \omega(t)} d t\right|:|z|=p, \omega \in \mathcal{B}\right\} .
\end{aligned}
$$

Now, let

and consider

$$
a_{2}=\frac{1}{z_{0}}-\lambda \int_{0}^{z_{0}} \frac{\left(1-|a|^{2}\right) \omega_{0}(t)}{1+\bar{a} t^{2} \omega_{0}(t)} d t
$$

$$
f_{0}(z)=\frac{z}{1-a_{2} z-\lambda z \int_{0}^{z} \frac{\left(1-|a|^{2}\right) \omega_{0}(t)}{1+\bar{a} t^{2} \omega_{0}(t)} d t} .
$$

The function $f_{0}$ shows that the above estimate is sharp.

Problem 3. Calculate the above maximum.

Remark 4. Let $f(z)=z / g_{\omega}(z) \in \mathcal{U}(\lambda, \mu)$. See (4). Since for any $\omega \in \mathcal{B}$ there exists a positive constant $C$ such that

$$
\left|g_{\omega}\left(z_{1}\right)-g_{\omega}\left(z_{2}\right)\right| \leq C\left|z_{1}-z_{2}\right|, z_{1}, z_{2} \in \mathbb{D},
$$

the function $g_{\omega}$ is uniformly continuous in $\mathbb{D}$. Therefore, it has a continuous extension to $\overline{\mathbb{D}}$. This fact implies that $f$ has a continuous extension to $\overline{\mathbb{D}} \backslash\left\{z: g_{\omega}(z)=0\right\}$. Hence, it makes sense to ask for the univalence of this continuous extension. From 
the proof of Theorem 2, it is obvious that this extension is univalent if $|1-\mu|<$ $\lambda \leq 1 / 2$ or if for $\lambda \in(1 / 2,1]$ the strict inequality $|1-\mu|<1-\lambda$ is valid.

On the other hand, Theorems 1 and 2 have the consequence that for $\lambda \in(1 / 2,1]$ the classes $\mathcal{U}(\lambda, \lambda)$ contain the interesting univalent slit mappings

$$
f(z)=\frac{z}{1-z\left(\frac{1}{p}+\lambda \int_{0}^{p} \frac{\left(1-a^{2}\right) d t}{1-a t^{2}}\right)+\lambda z \int_{0}^{z} \frac{\left(1-a^{2}\right) d t}{1-a t^{2}}}, a=\frac{1-\lambda}{\lambda} .
$$

These functions have a pole at $z=p$, and their derivatives vanish at $z=1$ and $z=-1$. We conjecture that possibly these classes and those functions deserve further research. Much of the investigations carried out in $[5,6,7,8,9,10]$ on $\mathcal{U}(\lambda)$ and some other related classes could be considered for further research with an aim to obtain meromorphic analogue of these classes.

Acknowledgments. The authors thank the referee for useful comments. The first author acknowledged the support from a USM research university grant 1001.PMATHS.8011038. The work of the second author is supported by Mathematical Research Impact Centric Support of Department of Science and Technology (DST), India (MTR/2017/000367).

\section{REFERENCES}

1. L. A. Aksentév, Sufficient conditions for univalence of regular functions (Russian), Izv. Vyš̌s. Učebn. Zaved. Matematika 1958 (4) (1958), 3-7.

2. L. A. Aksentév And F. G. Avhadiev, A certain class of univalent functions (Russian), Izv. Vyš̌. Učebn. Zaved. Matematika 1970(10) (1970), 12-20.

3. P. L. Duren, Univalent functions, Springer-Verlag, 1983.

4. A. W. Goodman, Univalent functions, Vols. 1-2, Mariner, Tampa, Florida, 1983.

5. M. Obradović, S. Ponnusamy, and K.-J. Wirths, Geometric studies on the class $\mathcal{U}(\lambda)$, Bull. Malaysian Math. Sci. Soc. 39(3) (2016), 1259-1284.

6. M. Obradović, S. Ponnusamy, and K.-J. Wirths, On relations between the classes $\mathcal{S}$ and $\mathcal{U}$, J. Analysis, 24 (2016), 83-93.

7. M. Obradović, S. Ponnusamy, and K.-J. Wirths, Logarithmic coefficients and a coefficient conjecture of univalent functions, Monatsh. Math. 185(3) (2018), 489-501.

8. S. Ponnusamy and K.-J. Wirths, Elementary considerations for classes of meromorphic univalent functions, Lobachevskii J. Math. 39(5) (2018), 713-716.

9. S. Ponnusamy and K.-J. Wirths, Coefficient problems on the class $U(\lambda)$, Probl. Anal. Issues Anal. 7(25), No. 1, (2018), 87-103.

10. A. Vasudevarao and H. Yanagihara, On the growth of analytic functions in the class $\mathcal{U}(\lambda)$, Comput. Methods Funct. Theory 13 (2013), 613-634.

S. K. Lee, School of Mathematical Sciences, Universiti Sains Malaysia, 11800 USM Penang, Malaysia.

E-mail address: sklee@usm.my

S. Ponnusamy, Department of Mathematics, Indian Institute of Technology Madras, Chennai-600 036, India.

E-mail address: samy@itm.ac.in

K.-J. Wirths, Institut für Analysis und Algebra, TU Braunschweig, 38106 BraunSchweig, Germany.

E-mail address: kjwirths@tu-bs.de 